\title{
Value of Preoperative Tissue Doppler Imaging-Derived Atrial Conduction Time as a Predictor of Postoperative Atrial Fibrillation in Patients Undergoing Mitral Valve Surgery for Mitral Valve Regurgitation
}

\author{
Hany Hassan Ebaid ${ }^{\text {a }}$, Ayman M. Hamoda ${ }^{\text {b }}$, Khaled E. Ahmed ${ }^{\text {a }}$, Sahar Abd Elaal Abd Allah ${ }^{\text {a }}$
}

\begin{abstract}
a
Department of cardiology, Benha faculty of medicine, Benha University, Egypt. b Department of cardiology, Mahalla Cardiac Center, Mahalla, Egypt
\end{abstract}

Correspondence to: Ayman M. Hamoda, Department of cardiology, Mahalla Cardiac Center, Mahalla, Egypt

Email:

ayman_hamoda1@hotmail.com

Received: 17 September 2020

Accepted: 15 November 2020

\section{Abstract:}

Objective: the study aimed to evaluate the value of the efficacy of the PA-TDI duration for predicting Perioperative atrial fibrillation in patients undergoing mitral valve surgery for mitral valve regurgitation. Methods: clinical and laboratory data of 100 patients who have mitral regurgitation and are planned for undergoing mitral valve surgery were collected and analyzed. Along with the usual echocardiographic parameters, PA interval measured by tissue Doppler was measured for all patients. Post-operatively, patients were followed up to detect occurrence of post-operative atrial fibrillation for one week postsurgery. Patients were divided into two groups according to incidence of POAF. The two groups were compared and PA-TDI was tested to assess its ability to predict POAF. Results: PA-TDI duration in POAF group was longer than in NO POAF group with highly significant difference between the two groups with P-value $<0.001$. It was found that PA-TDI duration $(\geq 151 \mathrm{~ms})$ has an accuracy of $66 \%$ in detection of $\mathrm{AF}$ with sensitivity $65.1 \%$ and specificity $67.6 \%$. Furthermore, positive predictive value for PA-TDI duration $(\geq 151 \mathrm{~ms}$ ) was $77.4 \%$ and its negative predictive value was 53.2\%. This study revealed that Pre-operative PA-TDI duration is the only independent predictor for POAF in this study. Conclusion: PA interval is a parameter which could be measured using tissue Doppler that does not require any additional expense and is a readily available. Consequently, it can help to identify individuals at high risk for PAOF who might need a more aggressive prophylactic and therapeutic approach and closer clinical follow up.

Key words: Cardiac surgery- mitral regurge - post-operative atrial fibrillation -TDI-PA intervaltotal atrial conduction time. 



\section{Introduction}

Postoperative atrial fibrillation (POAF) is the most imperative type of secondary AF. POAF is one of the complications in about one-third of patients undergoing cardiac surgery, resulting in considerable rises in hospital expenses, intensive care unit admission and hospital stay.

The prevalence of POAF is still substantial as well as troublesome (1). The etiology and risk factors for POAF are poorly understood, but advanced age, pre-existing cardiac conditions that cause rearrangement, and susceptibility towards inflammation have been consistently linked with POAF (2).

Several studies achieved much better prediction of POAF by measuring left atrial (LA) characteristics such as LA volume index (LAVI), LA strain and total atrial conduction time (TACT). TACT is theoretically measured on electrocardiography (ECG), but there are several alternative methods for measuring TACT.

The time interval from P-wave onset on ECG to the peak of the $A^{\prime}$ lateral wave on tissue Doppler imaging (TDI; PA-TDI duration) provides a reliable estimation of TACT, and PA-TDI duration has been shown to be significantly related to new-onset AF (3).

\section{Patients and methods}

This study is a prospective study that was conducted at Mahalla cardiac center and Benha university hospital for 100 patients who have mitral regurgitation and are eligible for undergoing mitral valve surgery during the period from January 2018 to August 2018 after approval of the ethical committee in Benha faculty of medicine and obtaining the patients informed consent.

Patients with significant valvar heart disease other than mitral regurge, who have chronic AF, who need emergency surgical procedure or patients with prior implantation of a permanent pacemaker were excluded from the study.

All patients were subjected to full history, thorough physical examination, resting 12 leads ECG \& routine laboratory investigations.

\section{- Transthoracic echocardiography :}

oLeft ventricular (LV) diameter, wall thickness, mitral valve inflow pattern $(\mathrm{E}$ wave, A wave, E wave deceleration time, $\mathrm{E} / \mathrm{A}$ ratio), LV ejection fraction, LA volumes and LAVI, LV mass and MR severity.

o A Tissue Doppler imaging (TDI) to assess PA-TDI interval, which is the time interval from the P-wave onset (lead II) to the peak of the $A^{\prime}$ wave on the tissue Doppler 
tracing of the LA lateral wall, and was measured in three cardiac cycles and averaged. Evaluation of transthoracic echocardiography was performed less than one month prior to the surgical intervention.

- All patients were monitored with continuous ECG monitoring for at least one week post-operative. A patient was determined to have AF when any episode lasted $>5$ minutes.

According to incidence of POAF, patients were divided into two groups: Group I: included patients who developed POAF \& Group II: included patients who did not develop POAF

Preoperatively, all cardiac drugs, including antiarrhythmic medication as $\beta$-blockers, calcium channel antagonists, angiotensinconverting enzyme inhibitors and angiotensin receptor blockers were continued until one day before the planned surgery.

The total fluid input (volume-in) and total fluid output (volume-out) were documented, and net fluid balance (NFB) was determined by subtracting the total fluid output from the total fluid input during the surgery and the following three days.

\section{Statistical analysis}

All data were collected, tabulated and statistically analyzed using STATA/SE version 11.2 for Windows (STATA Corporation, College Station, Texas).

After the calculation of each of the test statistics, the corresponding distribution tables were consulted to get the "P" (probability value). Statistical significance was accepted at $\mathrm{P}$ value $<0.05(\mathrm{~S})$ and a $\mathrm{P}$ value $<0.001$ was considered highly significant (HS). While, a $\mathrm{P}$ value $>0.05$ was considered non-significant. (4)

\section{Results}

The study included 100 patients, 33 patients were females (33\%) and 67 patients were males (67\%).Their age ranged from 38-75 years with mean age (56.9 \pm 7.8$)$ years , 52 patients were hypertensive (52 \%), 30 patients were diabetic (30\%), 37 patients were dyslipidemic (37\%), 7 patients had COPD (7\%) and 28 patients had a history of paroxysmal atrial fibrillation (28\%).

During the post-operative period (one week post-operative) 63 patients $(63 \%)$ developed AF (group I), 37 patients (37\%) remained in sinus rhythm (group II).

The two groups were comparatively matched with no significant differences regarding the demographic data or co morbidities in the two groups (Table 1)

\section{Preoperative Data:}

Mitral regurgitation in group I was degenerative, rheumatic and infective in [24 
$(38.1 \%), 37(58.7 \%), 2(3.2 \%)]$ while in group II, it was degenerative, rheumatic and infective in $[12(32.4 \%), 23(62.2 \%), 2$ $(5.4 \%) \& 3(8.1 \%)]$ respectively with no significant difference between the study groups. P wave duration did not differ significantly between the study groups $(118.8 \pm 10.5$ in group $I \& 116.9 \pm 14.8$ in group II, P-value 0.457) (Table 2).

A wave velocity in group I was $66.3 \pm 22.0$ and it was $77.7 \pm 26.5$ in group II; P-value 0.023. PA-TDI duration was $159.7 \pm 22.8$ in group I and $141.3 \pm 15.7$ in group II with Pvalue $<0.001$ (Tables 3).

\section{Operative data:}

There was no significant difference between studied groups regarding type of mitral valve intervention including replacement with metallic or bio-prosthetic valve $(\mathrm{P}$-value $=$ 0.941), bleeding volume in groups I and II $(1182.8 \quad \pm \quad 595.7 \quad \& \quad 1237.8 \quad \pm \quad 609.7$ respectively, $\mathrm{P}$-value $=0.660)$, concomitant tricuspid annuloplasty and incidence of POAF as patients who had undergone tricuspid annuloplasty $6.3 \%$ in group I and $10.8 \%$ in group II with P-value of 0.46
(Table 4).

\section{Post-operative data:}

There was no significant difference in Pwave duration on ECG between the patients with and without POAF $(110.1 \pm 16.0 \&$ $109.5 \pm 13.6$ respectively; P-value $=0.843$ ) (Table 5). ROC curve analysis (The receiveroperator characteristic) stated that the area under the curve for PA-TDI duration was 0.740 (95\% CI: $0.629-0.822 ; \mathrm{P}<0.0001)$. It was noted that PA-TDI duration $(\geq 151 \mathrm{~ms})$ has an accuracy of $66 \%$ in detection of $\mathrm{AF}$ with sensitivity equal to 65.1 and specificity $67.6 \%$ with positive predictive value $77.4 \%$ and negative predictive value was $53.2 \%$ (Table 6).

Univariate analysis of risk factors for predicting POAF showed that Duration of MR (P 0.023), A wave (P 0.026) and PATDI duration $(\mathrm{P}<0.001)$ were significant independent predictors of POAF (Table 7). Multivariate logistic regression analysis for predictors of POAF revealed that Preoperative PA-TDI duration ( $\mathrm{P}$ 0.001) is the only independent predictor for POAF in this study (Table 8). 
Benha medical journal vol.38, issue 2, 2021

Table (1): Demographic data

\begin{tabular}{lccc}
\hline Demographic data & Group I & Group II & P-value \\
\hline Male & 63 & 37 & \\
Female & $45(71.4 \%)$ & $22(59.5 \%)$ & 0.219 \\
Age (years) & $18(28.6 \%)$ & $15(40.5 \%)$ & \\
BSA $\left(\mathbf{m}^{2}\right)$ & $57.3 \pm 12.4$ & $54.5 \pm 14.0$ & 0.308 \\
DM & $1.62 \pm 0.17$ & $1.60 \pm 0.19$ & 0.761 \\
HTN & $22(34.9 \%)$ & $8(21.6 \%)$ & 0.161 \\
Dyslipidemia & $33(52.4 \%)$ & $19(51.4 \%)$ & 0.921 \\
COPD & $26(41.3 \%)$ & $11(29.7 \%)$ & 0.248 \\
PAF & $3(4.8 \%)$ & $4(10.8 \%)$ & 0.418 \\
\hline
\end{tabular}

Table (2): Pre-operative data

\begin{tabular}{lccc}
\hline Pre-operative data & GROUP I & GROUP II & P-value \\
\hline Etiology of MR & 63 & 37 & \\
Degenerative & & & 0.893 \\
Rheumatic & $24(38.1 \%)$ & $12(32.4 \%)$ & \\
Infective & $37(58.7 \%)$ & $23(62.2 \%)$ & \\
Duration of MR (years) & $2(3.2 \%)$ & $2(5.4 \%)$ & 0.019 \\
Creatinine (mg/dl) & $3.0 \pm 1.4$ & $2.4 \pm 1.1$ & 0.915 \\
P-wave duration (msec) & $1.6 \pm 0.9$ & $1.5 \pm 0.8$ & 0.457 \\
\hline
\end{tabular}


Table (3): Pre-operative echocardiographic data.

\begin{tabular}{|c|c|c|c|}
\hline $\begin{array}{l}\text { Echocardiographic } \\
\text { dimensions and volumes }\end{array}$ & Group I & Group II & P-value \\
\hline & 63 & 37 & \\
\hline LA dimension (mm) & $43.2 \pm 6.1$ & $44.3 \pm 5.6$ & 0.373 \\
\hline LA volume (ml) & $93.0 \pm 20.5$ & $87.8 \pm 10.4$ & 0.155 \\
\hline LAVI (ml/m2) & $58.1 \pm 14.0$ & $55.4 \pm 8.9$ & 0.292 \\
\hline EF by m-mode (\%) & $67.1 \pm 11.2$ & $70.1 \pm 9.1$ & 0.156 \\
\hline EF by Simpson's (\%) & $56.0 \pm 5.5$ & $57.5 \pm 4.5$ & 0.183 \\
\hline E wave $(\mathrm{cm} / \mathrm{s})$ & $106.4 \pm 25.8$ & $113.9 \pm 29.3$ & 0.186 \\
\hline A wave $(\mathrm{cm} / \mathrm{s})$ & $66.3 \pm 22.0$ & $77.7 \pm 26.5$ & 0.023 \\
\hline $\mathbf{E} / \mathbf{A}$ & $1.85 \pm 0.89$ & $1.84 \pm 1.47$ & 0.954 \\
\hline Deceleration time (ms) & $209.1 \pm 67.0$ & $203.2 \pm 62.6$ & 0.667 \\
\hline$A^{\prime}$ wave peak $(\mathrm{cm} / \mathrm{s})$ & $9.0 \pm 3.9$ & $8.3 \pm 2.9$ & 0.360 \\
\hline e' septal $(\mathrm{cm} / \mathrm{s})$ & $8.4 \pm 3.1$ & $8.6 \pm 3.6$ & 0.811 \\
\hline e' lateral $(\mathrm{cm} / \mathrm{s})$ & $10.2 \pm 3.1$ & $10.2 \pm 3.8$ & 0.934 \\
\hline E/e' (septal) & $14.1 \pm 5.1$ & $15.7 \pm 7.7$ & 0.189 \\
\hline E/e' (lateral) & $11.2 \pm 3.5$ & $12.6 \pm 5.0$ & 0.102 \\
\hline \multicolumn{4}{|l|}{$\underline{\text { TR grade }}$} \\
\hline Grade 0 ; & $8(12.7 \%)$ & $2(5.4 \%)$ & 0.944 \\
\hline Grade 1 & $39(61.9 \%)$ & $28(75.7 \%)$ & \\
\hline Grade 2 & $16(25.4 \%)$ & $7(18.9 \%)$ & \\
\hline TR maximum PG (mmHg) & $33.7 \pm 12.4$ & $37.9 \pm 15.1$ & 0.131 \\
\hline PA-TDI duration (ms) & $159.7 \pm 22.8$ & $141.3 \pm 15.7$ & $<0.001$ \\
\hline
\end{tabular}


Benha medical journal vol.38, issue 2, 2021

Table (4): Operative data.

\begin{tabular}{lccc}
\hline Operative data & GROUP I & GROUP II & P-value \\
\hline Type of MV repair & 63 & 37 & \\
Metallic & $45(71.4 \%)$ & $25(67.6 \%)$ & 0.941 \\
Bioprosthetic & $18(28.6 \%)$ & $12(32.4 \%)$ & \\
Tricuspid annuloplasty & & & 0.463 \\
& $4(6.3 \%)$ & $4(10.8 \%)$ & \\
\hline
\end{tabular}

Table (5): Post-operative data.

\begin{tabular}{lccc}
\hline Post-operative data & GROUP I & GROUP II & P-value \\
\hline Post-operative medications & $\mathbf{6 3}$ & 37 & \\
Dopamine & $33(52.4 \%)$ & $25(67.6 \%)$ & 0.137 \\
Dobutamine & $22(34.9 \%)$ & $7(18.9 \%)$ & 0.089 \\
Adrenaline & $8(12.7 \%)$ & $5(13.5 \%)$ & 1.000 \\
B-blockers & $37(58.7 \%)$ & $28(75.7 \%)$ & 0.086 \\
Amiodarone & $28(44.4 \%)$ & $12(32.4 \%)$ & 0.161 \\
\hline
\end{tabular}

Table (6): the predictive performance of pre-operative PA-TDI duration for POAF; ROC curve analysis

\begin{tabular}{|c|c|c|c|c|c|c|c|}
\hline $\begin{array}{l}\text { Cut-off } \\
\text { value }\end{array}$ & $\begin{array}{c}\text { Sensitivity } \\
\% \\
(95 \% \mathrm{CI}) \\
\end{array}$ & $\begin{array}{c}\text { Specificity } \\
\% \\
(95 \% \text { CI }) \\
\end{array}$ & $\begin{array}{l}\text { PPV \% } \\
(95 \% \mathrm{CI})\end{array}$ & $\begin{array}{c}\text { NPV \% } \\
(95 \% \text { CI })\end{array}$ & $\begin{array}{c}\text { Accuracy } \\
\% \\
(95 \% \text { CI }) \\
\end{array}$ & $\begin{array}{l}\text { AUROC } \\
(95 \% \mathrm{CI})\end{array}$ & $\begin{array}{c}\text { P- } \\
\text { value }\end{array}$ \\
\hline $\begin{array}{l}\text { PA-TDI } \\
\text { duration } \geq \\
151 \mathrm{~ms}\end{array}$ & $\begin{array}{c}65.1 \% \\
(52.0-76.7)\end{array}$ & $\begin{array}{c}67.6 \% \\
(50.2-82.0)\end{array}$ & $\begin{array}{c}77.4 \% \\
(63.8-87.7)\end{array}$ & $\begin{array}{c}53.2 \% \\
(38.1-67.9)\end{array}$ & $\begin{array}{c}66 \% \\
(55.9-75.2)\end{array}$ & $\begin{array}{c}0.740 \\
(0.629-0.822)\end{array}$ & $<0.001$ \\
\hline
\end{tabular}

(ROC curve: Receiver Operating Characteristic curve, PPV: Positive Predictive Value, NPV: Negative Predictive Value, AUROC: Area under ROC, CI: Confidence Interval) 


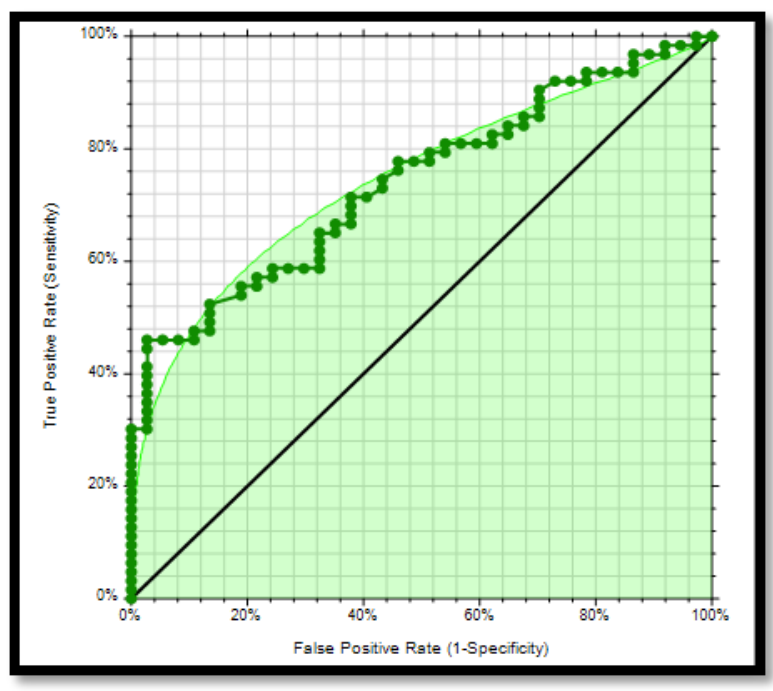

Figure (1): ROC curve analysis pre-operative PA-TDI duration in predicting POAF

Table 7: Univariate analysis for predictors of POAF.

\begin{tabular}{lcc}
\hline & Unadjusted OR & P value \\
\hline Duration of MR (years) & 1.474 & 0.023 \\
A wave (cm/s) & 0.980 & 0.026 \\
Pre-operative PA-TDI duration (ms) & 0.980 & $<0.001$ \\
\hline
\end{tabular}

Table 8: Multivariate analysis for predictors of POAF.

\begin{tabular}{lcc}
\hline & Adjusted OR & P value \\
\hline Duration of MR (years) & 1.328 & 0.143 \\
A wave (cm/s) & 0.984 & 0.101 \\
Pre-operative PA-TDI duration (ms) & 1.046 & 0.001 \\
\hline
\end{tabular}

\section{Discussion}

Despite POAF was thought to be transient and self-limiting, it is recently appreciated as a risk factor for stroke and mortality (5). In the present study, Patients' age in the two groups was slightly higher in group I with no significant difference between the two groups as regard gender distribution (P-value $=0.219$ ). Takahashi et al study (6) showed no significant difference between the mean age in the two groups $(59.4 \pm 16.6$ in NO POAF 
group vs $65.8 \pm 11.3$ in the POAF group with P-value 0.05) with no significant gender changes. Also Iliescu et al study (8) and Filardo et al study (7) showed no significant difference regarding gender distribution between POAF group and sinus group.

Regarding etiology of mitral regurgitation in group I, it was degenerative, rheumatic and infective in $[24(38.1 \%), 37(58.7 \%), 2$ (3.2\%)] respectively. In group II, it was degenerative, rheumatic and infective in [12 (32.4\%), $23(62.2 \%), 2$ (5.4\%) \&3 (8.1\%)] respectively with no significant difference between the study groups.

This is discordant with Takahashi et al study (6) in which degenerative etiology was the highest etiology for mitral regurgitation with $48 \%$ in sinus group and $82 \%$ in POAF group with $\mathrm{P}$-value $=0.004$. This disagreement is mostly due to different etiology of valve affection in our study because of endemic incidence for rheumatic fever.

P-wave duration did not differ significantly, in the present study, between the study groups. This was concordant with Takahashi et al study (6) where P-wave duration on surface ECG was not statistically different between the two groups (P-value 0.3295). On the contrary, a prospective cohort study included 299 patients undergoing CABG stated that patients with POAF had a mean P-wave duration of $110.32 \pm 13.13$ and patients with no POAF $102.28 \pm 11.30$ with P-value $<0.001(\mathbf{1 0})$.

Furthermore, another previous study has indicated that $\mathrm{P}$-wave duration and dispersion were shorter significantly in patients with POAF due to the elevated B-adrenergic tone. Changes in P-wave characteristics can be attributed to atrial stretch and fluid overload. P-wave duration and dispersion can be decreased by fluid diuresis, which may be the cause of these different results from various studies (11).

Pre-operative A wave velocity differed significantly in the current study. This was concordant with Iribarren et al study (12), which showed no significant difference between the POAF and NO POAF group regarding echocardiographic parameters apart from lateral mitral annulus a wave, which was higher in the NO POAF group ( $\mathrm{P}$ - value 0.01). Also Takahashi et al study (6) reported that there was statistically significant difference between the POAF and NO POAF group regarding the A wave peak velocity.

In our study, PA-TDI duration was $159.7 \pm$ 22.8 in group I and $141.3 \pm 15.7$ in group II with P-value <0.001. Müller et al study (9) revealed that patients with POAF after cardiac surgery had a significantly longer PA-TDI interval pre-operatively $(\mathrm{P}<0.001)$. Also 
Takahashi et al study (6) stated that POAF was associated with longer PA-TDI duration (P-value 0.0006).

Our study found that PA-TDI duration $(\geq 151$ msec) has an accuracy of $66 \%$ in detection of AF with sensitivity $65.1 \%$, specificity of $67.6 \%$, positive predictive value $77.4 \%$ and negative predictive value $53.2 \%$. This result was relatively concordant with Takahashi et al study (6) where the optimal P-A' interval duration was $159.4 \mathrm{msec}$ with sensitivity of $55.8 \%$ and specificity of $84.6 \%$.

Our results showed that there was no significant difference regarding concomitant tricuspid annuloplasty and incidence of POAF. This is concordant with Bramer et al study(13) results where tricuspid valve surgery was done for $11.9 \%$ of the patients in the NO POAF group and $13.9 \%$ in the POAF group $(\mathrm{P}-\mathrm{value}=0.40)$. Candan et al study (14) reported that tricuspid valve surgery was done for $21.1 \%$ of the patients in the NO POAF group and $33.3 \%$ in the POAF group (P-value 0.48).

The use of post-operative inotropes (Dopamine, Dobutamine, Adrenaline) did not differ statistically between the study groups ( $\mathrm{P}$-value >0.05). This result is in agreement with Takahashi et al study (6) results where administration of inotropes was not associated with POAF. This was discordant with Maesen et al study (15) result where dopamine and dobutamine use are associated with a higher occurrence of POAF.

In the present study, Post-operative antiarrhythmic drugs (beta blockers and amiodarone) did not differ significantly between the study groups (P-value >0.05). This result was concordant with Takahashi et al study (6) where using beta blockers did not affect POAF incidence. This was discordant with Maesen et al study (15) results as patients receiving $\beta$-blockers post-operatively have fewer episodes of AF. According to the ESC guidelines 2016, $\beta$-Blocker prophylaxis is the best-established preventive therapy and should be started or continued before cardiac surgery (16).

\section{Conclusion}

PA interval measured by TDI preoperatively seems to be a simple method to predict incidence of POAF in patients undergoing mitral valve replacement and it may be part of preoperative cardiovascular examination. Our study suggests that longer PA-TDI duration to be additive to conventional risk factors and biomarkers in predicting POAF and that PATDI duration is an independent predictor of POAF and the best cut off value of PA-TDI duration for POAF is $151 \mathrm{msec}$. PA interval is a parameter, a readily available \& easily 
done marker, which can help to simply identify individuals at high risk for PAOF who might need a more aggressive prophylactic and therapeutic approach and closer clinical follow up.

\section{Limitation}

The relatively limited number of the patients and only two center experience may limit our results.

\section{References}

1- Dobrev D, Aguilar M, Heijman J, Jean-Baptiste, Guichard and Nattel S. Postoperative atrial fibrillation: mechanisms, manifestations and management. Nature Reviews Cardiology 2019;16:417-35.

2- Zakkar M, Ascione R, James AF, Angelini GD, Suleiman MS. Inflammation, oxidative stress and postoperative atrial fibrillation in cardiac surgery. Pharmacol Ther 2015;154:13-20.

3- de Vos CB, Weijs G, Crijns HJGM, Cheriex EC, Palmans A, Habets J, et al. Atrial tissue Doppler imaging for prediction of new onset atrial fibrillation. Heart 2009; 95: 835 - 40 .

4- Altman DG and Bland JM (1983): Measurement in Medicine: The Analysis of Method Comparison Studies. The Statistician 32:307-17.

5- Lin MH, Kamel H, Singer DE, Wu YL, Lee M, Ovbiagele B. Perioperative/Postoperative Atrial Fibrillation and Risk of Subsequent Stroke and/or Mortality. Stroke 2019:50:1364-71.

6- Takahashi S, Katayama K, Watanabe M, Kodama H, Taguchi T, Kurosaki $\mathrm{T}$, et al. Preoperative Tissue Doppler Imaging-Derived Atrial Conduction Time Predicts Postoperative Atrial
Fibrillation in Patients Undergoing Mitral Valve Surgery for Mitral Valve Regurgitation. Circulation Journal 2016; 80(1):101-9.

7- Filardo G, Hamilton C, Hamman B, Hebeler R, Adams J and Grayburn p. New-onset Postoperative Atrial Fibrillation and Long-Term Survival after Aortic Valve Replacement Surgery. Ann Thorac Surg 2010;90:474-80

8- Iliescu A, Salaru D, Achitei I, Grecu M, Floria M, and Tinica G. Postoperative atrial fibrillation prediction following isolated surgical aortic valve replacement. Anatol J Cardiol. 2018; 19(6): 394400.

9- Müller P, Hars C, Schiedat F, Bösche LI, Gotzmann M, Strauch J, et al. Correlation Between Total Atrial Conduction Time Estimated via Tissue Doppler Imaging (PA-TDI Interval), Structural Atrial Remodeling and New-Onset of Atrial Fibrillation After Cardiac Surgery. J Cardiovasc Electrophysiol 2013;24: 626-631.

10- Fangqin Wu, Ying Wu, Wenyan Tao, Haibo Zhao and Dongyan Shen. Preoperative P-wave duration as a predictor of atrial fibrillation after coronary artery bypass grafting: A prospective cohort study with meta-analysis. International Journal of Nursing Sciences 2018;5:151-6

11- Chandy J, Nakai T, Lee RJ, Bellows WH, Dzankic $\mathrm{S}$, Leung JM. Increases in $\mathrm{P}$-wave dispersion predict postoperative atrial fibrillation after coronary artery bypass graft surgery. Anesth Analg. 2004;98(2):303-10.

12- Iribarren J, Jiménez J, Barragán A, Brouard M, Lacalzada L, Lorente 1 , et al. Left Atrial Dysfunction and New-Onset Atrial Fibrillation After Cardiac Surgery. Rev Esp Cardiol. 2009;62(7):774-80.

13- Bramer S, Straten A, Hamad M, Krista van den Broek K, Jos Maessen J, and Berreklouw E. NewOnset Postoperative Atrial Fibrillation Predicts 
Late Mortality After Mitral Valve Surgery. Ann

Thorac Surg 2011;92:2091-6.

14- Candan O, Ozdemir N, Aung SM, Dogan C, Karabay CY, Gecmen C, et al. Left Atrial Longitudinal Strain Parameters Predict Postoperative Persistent Atrial Fibrillation Following Mitral Valve Surgery: A Speckle Tracking echocardiography Study. Echocardiography 2013;30(9): 1061 -8.
15- Maesen Bart, Nijs Jan, Maessen Jos, Allessie Maurits, Schotten Ulrich. Post-operative atrial fibrillation: a maze of mechanisms. Europace. 2012; 14(2): 159-74.

16- Kirchhof P, Benussi S, Kotecha D, Ahlsson A, Atar D, Casadei B, et al. 2016 ESC Guidelines for the management of atrial fibrillation developed in collaboration with EACTS. European Heart Journal (2016) 37, 28

To cite this article: Hany Hassan Ebaid, Ayman M. Hamoda, Khaled E. Ahmed , Sahar Abd Elaal Abd Allah. Value of Preoperative Tissue Doppler Imaging-Derived Atrial Conduction Time as a Predictor of Postoperative Atrial Fibrillation in Patients Undergoing Mitral Valve Surgery for Mitral Valve Regurgitation. BMFJ 2021;38(2): 443-454, DOI: 10.21608/bmfj.2020.43298.1318 